\title{
ERIS, first generation becoming second generation, or re-vitalizing an AO instrument
}

\author{
A. Cortes ${ }^{\mathrm{a}}$, R. Davies ${ }^{\mathrm{a}}$, H. Feutchgruber ${ }^{\mathrm{a}}$, E. Sturm ${ }^{\mathrm{a}}$, M. Hartl ${ }^{\mathrm{a}}$, F. Eisenhauer ${ }^{\mathrm{a}}$, H. Huber ${ }^{\mathrm{a}}$, E. Wiezorrek ${ }^{\mathrm{a}}$, \\ M. Plattner ${ }^{\mathrm{a}}$, A. Buron ${ }^{\mathrm{a}}$, J. Schubert ${ }^{\mathrm{a}}$, S. Gillessen ${ }^{\mathrm{a}}$, C. Rau ${ }^{\mathrm{a}}$, N. Förster-Schreiber ${ }^{\mathrm{a}}$,

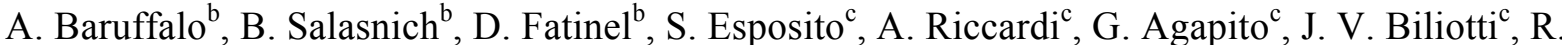

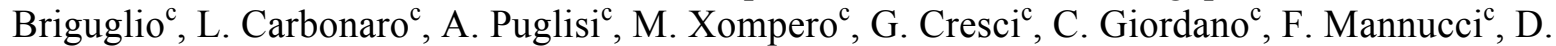

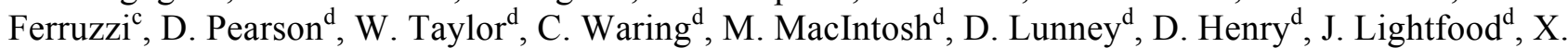

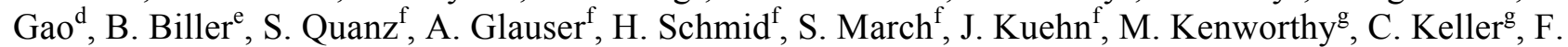

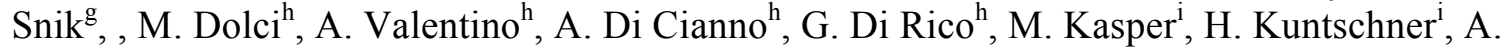 \\ Glindemann ${ }^{\mathrm{i}}, \mathrm{R}$. Dorn ${ }^{\mathrm{i}}, \mathrm{H}$. Jeroen ${ }^{\mathrm{i}}$ \\ ${ }^{a}$ Max-Plank-Institute für Extraterrestrische Physik, Giessenbachstrasse 1, 85748 Garching, Germany \\ ${ }^{\mathrm{b}}$ INAF-Osservatorio Astronomico di Padova, Vicolo Osservatorio 5-35122, Padova PD, Italy \\ ${ }^{c}$ INAF-Osservatorio Astrofisico di Arcetri, Largo E. Fermi 5, I-50125, Firenze, Italy \\ ${ }^{\mathrm{d}}$ UK Astronomy Technology Centre, STFC, Blackford Hill, Edinburgh, EH9 3HJ, UK \\ ${ }^{\mathrm{e}}$ Institute for Astronomy, Royal Observatory Edinburgh, Blackford Hill, Edinburgh, EH9 3HJ UK \\ ${ }_{\mathrm{f}}^{\mathrm{f}}$ TH Zürich, Institute for Particle Physics and Astrophysics, Wolfgang-Pauli-Strasse 27, CH-8093 \\ Zürich, Switzerland \\ ${ }^{g}$ Leiden Observatory, Leiden University, P.O. Box 9513, 2300 RA, Leiden, The Netherlands \\ ${ }^{\mathrm{h}}$ INAF-Osservatorio Astronomico di Terramo, Via Maggini snc, I-64100, Terramo, Italy \\ ${ }^{i}$ European Southern Observatory, Karl-Schwarzschild-Straße 2, 85748 Garching, Germany
}

\begin{abstract}
Within the VLT instrumentation program, the second generation instrument ERIS (Enhanced Resolution Imager and Spectrograph) combines two key scientifically successful elements of the VLT first generation instrumentation program: It consists of a full renovation of the integral field spectrograph SPIFFI and a new near-IR camera NIX, implementing the most scientifically important imaging modes offered so far by NACO (imaging in the J to M bands, astrometry, Sparse Aperture Masking and Apodizing Phase Plate (APP) coronagraphy). Both diffraction limited sub-systems of ERIS make use of the latest AO technologies with the newly installed AOF (AO Facility) Deformable Secondary Mirror with 1170 actuators and a new laser guide star system.

We will describe the changes that will be implemented, give a summary of what SINFONI is currently achieving, and present what to expect from the performance upgrade. With instruments becoming more complex and therefore increasing development times, we describe the challenges to improve image quality, spectral and spatial resolution on the same focus of a VLT UT, which could become valuable lessons for the extension of the life of actual instruments and of future ones. We will address the impact of the aging of the instrument and what critical parts to consider in the design in view of future upgrades, to possibly extend the performances, capabilities and lifetime at lower development costs.
\end{abstract}

Keywords: ERIS, Adaptive Optics, Instrumentation, Spectrograph, Imaging, Infrared, Deformable Secondary Mirror.

\section{INTRODUCTION}

ERIS will provide a diffraction limited near-infrared imaging and spectroscopic capability to observe several interesting astronomical objects, like interstellar gas, brown dwarfs, stars being born, planets and dust discs around other stars, high obscured astrophysical sources such as the Galactic Center and the centres of other galaxies, including active galactic nuclei are objects that are too cold to shine in visible wavelengths but become observable when viewed in the infrared. And its enhanced sensitivity, particularly in J-band (better throughput and better AO performance, with also better line spectral profiles) means that will be an excellent instrument for studying galaxy evolution at high redshift. 
This paper explains the current design and characteristics of ERIS, from its status at FDR (Final Design Review), i.e. with only some details that will be defined in the future and some small improvements that can be done.

ERIS, the Enhanced Resolution Imager and Spectrograph, as a new IR instrument for the VLT, is actually two instruments in one. But it can also be seen as the evolution of two of the best IR instruments at Paranal, which are at the end of their design lifetimes. Those instruments are SINFONI and NACO, which will be replaced respectively by ERIS-SPIFFIER and ERIS-NIX. One is the improved version of SPIFFI, with new optics, detector, and electronics. And NIX is a near-IR imaging camera that will provide the most important modes of NACO.

Both ERIS instruments will provide diffraction limited sources thanks to the use of the AOF infrastructure and the Deformable Secondary Mirror (DSM) on a narrow field of view (FoV) of 24" and 53" for NIX, and 8", 3.2" and 0.8" for SPIFFIER.

The ERIS design is modular and consists of the following main components (see Figure 1 and Figure 2):

- An infrared camera NIX with the detector operating at 40 Kelvin with a compressor for closed cycle cooler.

- $\quad$ The Integral Field Spectrograph SPIFFIER, corresponding to an upgraded version of SPIFFI.

- An AO module, which includes the dichroic beam splitter and the NIX selector mirror.

- The Calibration Unit, which includes the fold mirror that inserts the calibration light into the optical path.

- $\quad$ Three cabinets hosting the electronics of all system and subsystems.

- A central mechanical structure that host all instrument components, to be attached to the adaptor/rotator of the cassegrain focus on the UT4 telescope.

- $\quad$ Auxiliary components that include a cable wrap and an ERIS instrument handling tool.

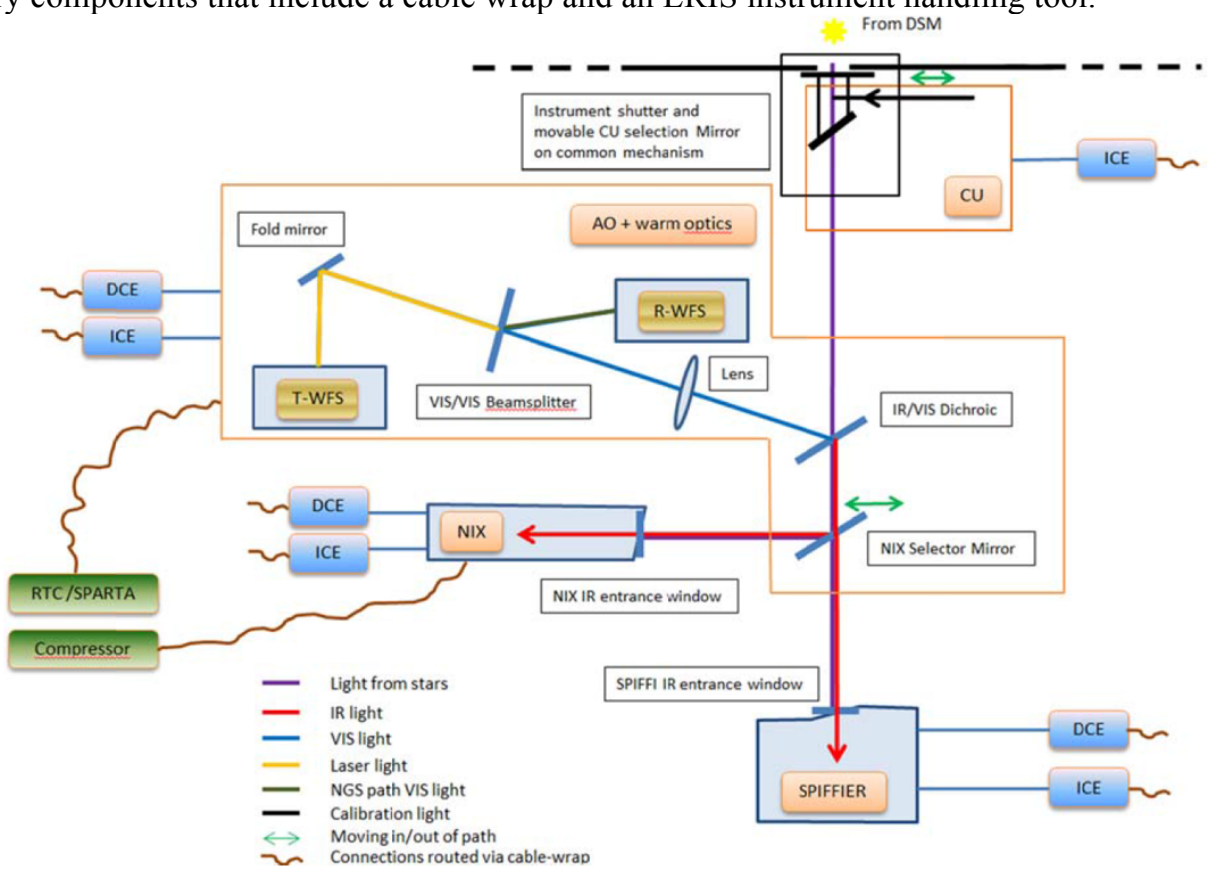

Figure 1. Simplified diagram of the different modules of ERIS and the optics concept to illustrate the light path from the entrance of the telescope and the calibration unit, to SPIFFIER or NIX.

The telescope DSM is the aperture stop. The telescope's focal plane is shifted by $500 \mathrm{~mm}$ compared to the VLT optical default configuration.

Both instruments will have scientific detectors Hawaii 2RG, that are going to be read by only one NGC, with the advantage of reducing cost, volume and weight. However, this configuration has the limitation that only one of the SPIFFIER or NIX detectors can be read at a time, but is not an issue as the instruments are not intended to be used in parallel. 


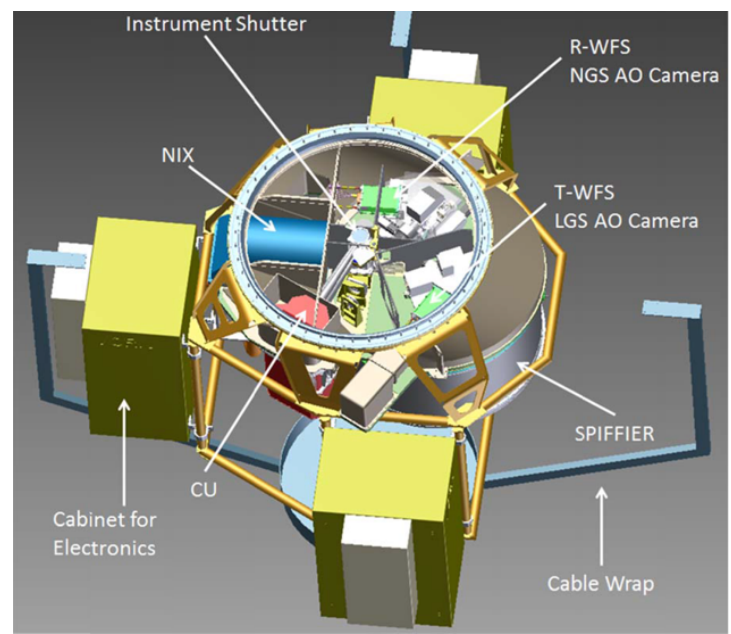

Figure 2. ERIS mechanical structure, here showing a top view of the NIX and SPIFFIER instruments, the AO module and the 3 cabinets. Preliminary CAD model.

\section{ADAPTIVE OPTICS (AO) MODULE}

The AO module uses the existing AOF sub-systems and components provided by ESO, that are installed already in the telescope and working since the end of 2016. The AO correction is provided by the AOF Deformable Secondary Mirror (DSM) and the artificial Laser Guide Star (LGS) is generated by the 4LGSF system.

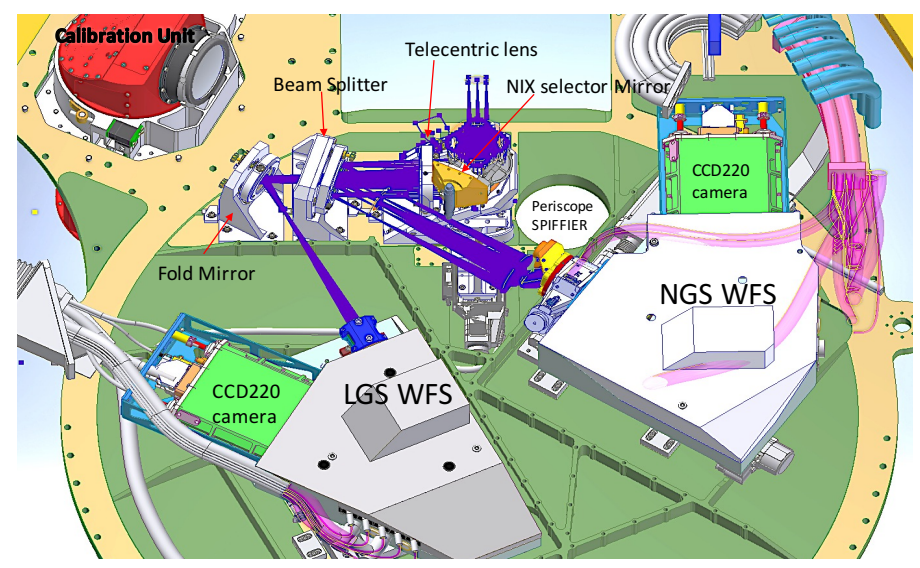

Figure 3. AO module, with the LGS and NGS WFS units mounted on the ERIS Optical Plate, the calibration unit and the hole for the Periscope with the window for SPIFFIER.

The AO WFSs opto-mechanical design has been constrained by the general design choice of using flat optics to relay the telescope beam to the AO WFSs to maximize the stability of Non-Common Path Aberrations (NCPA). As a direct consequence of this design choice, the selection of the Natural Guide Star (NGS) in the AO field is not performed by an optical field selector (tip-tilt mirror on a pupil image), but implementing each WFS units as a compact board supported by stages to scan the field (circular, about $32 \mathrm{~mm}$ radius). The same approach is adopted for tracking the focus in both NGS and LGS WFS case.

The distance between the Cassegrain Flange and the top of SPIFFIER provides another major space constraint to fit the Warm Optics (WO) design. This requires increasing the Cassegrain Back Focal Length (BFL) by $250 \mathrm{~mm}$ with respect to the nominal VLT one, resulting in a formal Change Request to ESO.

The AO module is fed by a dichroic beam-splitter which reflects the visible light and transmit the IR light to NIX and SPIFFIER. 


\subsection{Deformable Mirror}

The ERIS instrument will be installed at the cassegrain focus of UT4, and will be using the telescope DSM, that is already used by GRAAL and GALACSI by the ERIS AO module, to provide diffraction limited images to both NIX and SPIFFIER. The DSM provides wavefront correction at the frequency of $1 \mathrm{kHz}$. The DSM is a Zerodur mirror with dimensions of $1.12 \mathrm{~m}$ diameter and $2 \mathrm{~mm}$ thickness and with a convex hyperboloid shape. It has 1170 voice-coil actuators controlled in closed loop by co-located capacitive positions sensors, manufactured by Microgate s.r.1 and A.D.S. The mirror alignment to the telescope axis is provided by a hexapod that also allows the chopping and field stabilization. The DSM performs chopping through a tilt of the M2 in its plane, with a range from $0.1 \mathrm{~Hz}$ to $5 \mathrm{~Hz}$ depending on the specific observing program, and with a maximum chopping throw of $\pm 20 \operatorname{arcsec}$, including DC offset.

The specification for the total actuator stroke of the DSM is $\pm 40-50 \mu \mathrm{m}$ including tip-tilt, with a resolution of $5 \mathrm{~nm}$. The actuator hysteresis is less than $1 \%$ of the full stroke. The DSM surface stability in AO open loop is less than $20 \mathrm{~nm}$ RMS wavefront error over $8 \mathrm{hrs}$ ( or $5.6^{\circ} \mathrm{C}$ variation). The specification for the micro roughness is less than $2 \mathrm{~nm} \mathrm{rms}$.

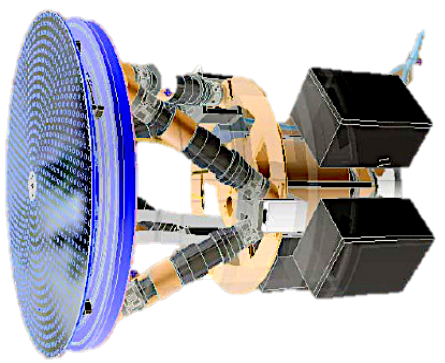

Figure 4. Deformable Secondary mirror (DSM) that will be used by ERIS for the AO module.

\subsection{Laser guide star facility (LGSF)}

ERIS will use one of the 4LGSF, which are part of the ESO Adaptive Optics Facility (AOF), placed in the side of the telescope primary mirror. The lasers are exciting the atomic sodium layer in the mesosphere $(\sim 90 \mathrm{~km})$ at the wavelength of 589.159nm, based on the ESO narrow-band Ramman optical Fiber Amplifier and a master oscillator power amplifier (MOPA) configuration patented technology, attached to a $30 \mathrm{~cm}$ launch telescope. The 4 LGS can operate at the same time, but they are independent.

The average power is $\geq 17 \mathrm{~W}$ after the launch telescope, and the optical throughput of each laser guide star unit is designed to be $>88 \%$. The laser beam propagated to the mesosphere shall be circularly polarized. The 4LGSF may receive from the client AO system the command to toggle on and off the $\mathrm{Na} \mathrm{D}^{2}$ line by $5 \mathrm{GHz}$, to tune or detune the sodium excitation.

As the LGS are launched from the side of the primary mirror, there is a small elongation of the spots on the WFS for the $8 \mathrm{~m}$ telescope. This can cause an error of the LGS centroid height estimation of $0.3 \mathrm{~nm}$ of focus wavefront error, but this is a negligible error that can be corrected with the use of a natural guide star.
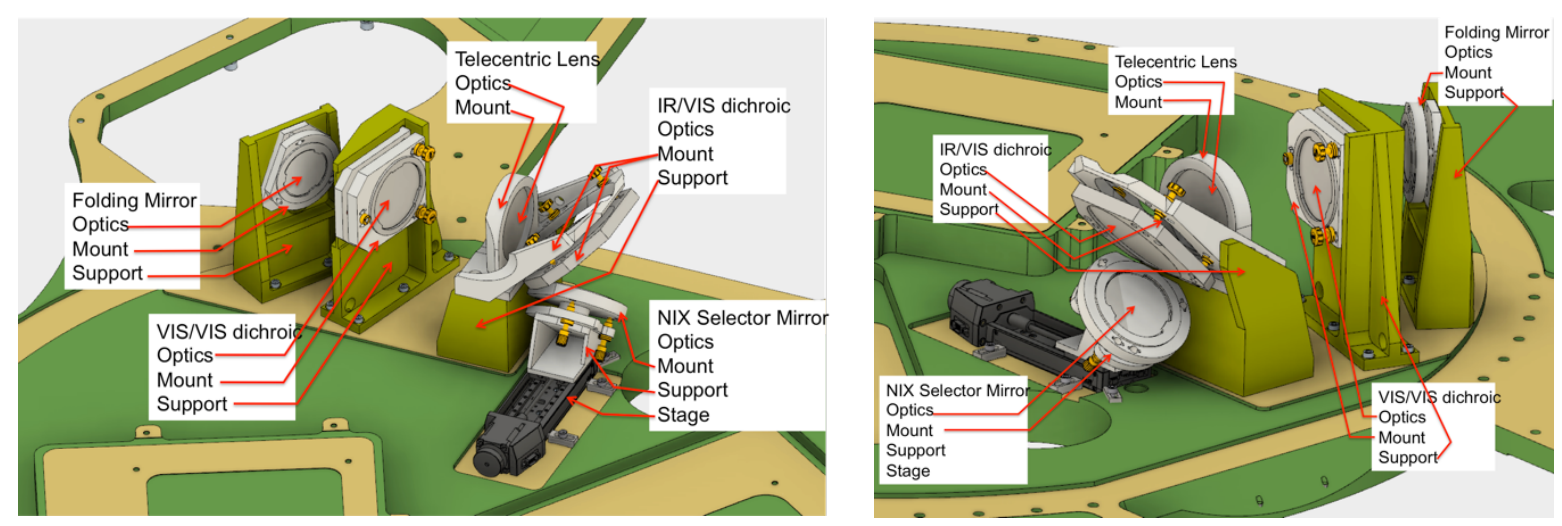

Figure 5. CAD design of the main Warm Optics components standing over the optical plate. 


\subsection{Warm Optics}

The Warm Optics (WO) corresponds to the optics required as relay from the telescope beam to the instruments (NIX or SPIFFIER) and to the AO WFS. A VIS/IR dichroic reflects the visible light from the telescope or the CU towards the AO sub-system and transmits the light longward of $1.08 \mu \mathrm{m}$ to the NIX camera or SPIFFIER spectrometer by a selector mirror that can be controlled remotely to switch the light beam.

The reflected light from the dichroic passes through a telecentric lens and is then split by a beam splitter into the reflected light for the NGS WFS and the transmitted light for the LGS WFS.

\subsection{LGS Wavefront Sensors}

For the LGS WFS the camera is provided with an internally integrated Shack-Hartmann with 40x40 lenslet array allocating 6x6 pixels per sub-aperture. The lenslet focal length provides a 5"x5" FoV per sub-aperture.

The LGS WFS beam comes from the WO dichroic, with a K-prism to derotate the pupil. Afterwards the pupil is stabilized against flexures and misalignments by a steering mirror. The CCD220 camera is mounted on the WFS stage and therefore moving together with it. The stage is used for tracking the best focus of the laser star when its height is changing with elevation and sodium profile evolution. A shutter can stop the incoming beam to protect the detector against overillumination and calibrate dark frames.

The LGS WFS has an Entrance Lens producing a F/20 beam and relaying the input telecentric focal plane to a tip-tilt mirror that stabilizes the lateral decentering of the pupil on the SH lenslet array. The main contributors to the stroke requirement of the mirror are the pupil plane lateral drifts due to alignment error between WFS and instrument rotator axis, $\mathrm{K}$-mirror and flexures.

A Pupil Rotator (the same as NGS WFS) allows to de-rotate the actuator pattern of the DSM on the SH lenslet array when ERIS instrument rotator is operating in field tracking mode.

The LGS WFS operates only on-axis, and therefore does not require stages to patrol the field. LGS on-axis location and stabilization are devoted to the 4LGSF launcher, providing an internal large stroke field steering mirror for LGS acquisition and drift tracking, and a low stroke fast TT corrector.
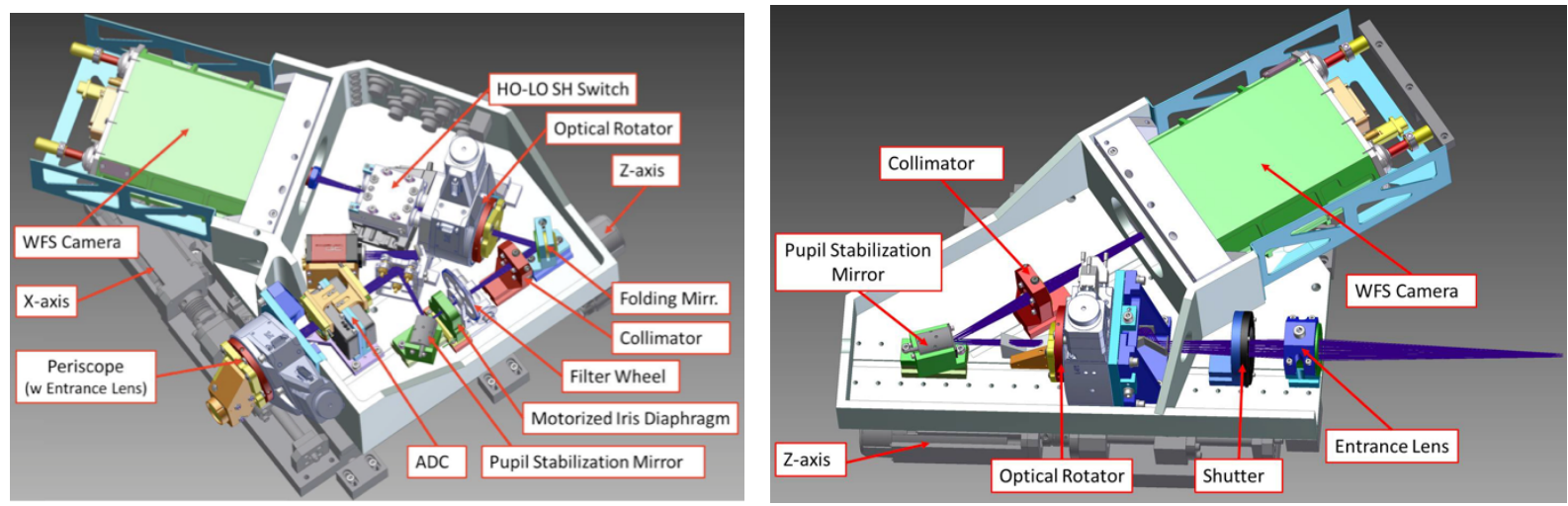

Figure 6. CAD design of the NGS WFS (left) and LGS WFS (right).

\subsection{NGS Wavefront Sensors}

For the NGS WFS, the same camera is provided but without the integrated lenslet array. It is a configurable ShackHartmann sensor with $40 \times 40$ and $4 \times 4$ subapertures, respectively for HO corrections (NGS operation) and LO \& truth sensing for LGS operation. The two configurations are called HO WFS and LO WFS and are selected by exchanging two lenslets arrays using a translation stage. The CCD220 camera is mounted on the WFS stage and therefore moving together with it. A 4-positions filter wheel is hosting a beam stop for dark calibration, a pass-all filter for normal operations and two filters to reduce the light level and avoid detector over-illumination.

It has a dual axis stage supporting the WFS board. One (focus) stage moves in the direction of the input optical axis, the other (X stage) in the orthogonal direction, parallel to the plane of the WFS board. 
A beam splitter dichroic transmits the wavelengths shorter than $600 \mathrm{~nm}$ to a technical camera that can be also used for NGS acquisition. The wavelengths in the range $600 \mathrm{~nm}-1000 \mathrm{~nm}$ are reflected toward the pupil stabilization mirror (PSM). The PSM on the F/20 focal plane is used to stabilize the lateral decentering of the pupil on the SH lenslet array.

The imaged beam from the WO dichroic is tracked by an optical pupil rotator (K-prism) that de-rotates the DSM actuator patter on the SH lenslet array when ERIS instrument rotator is operating in field tracking mode, and has an ADC that compensate of the differential atmospheric dispersion (down to $\mathrm{z}=70 \mathrm{deg}$ ).

All the components are installed on a support board, which is actively aligned with the entrance beam through a dual-axis stage (lateral and focus translation) of the NGS WFS unit.

\subsection{AO observing modes}

The AO module will support the following observing modes:

1. SCAO LGS mode with (any) one of the 4LGSF laser on-axis and one tilt star in the visible (lambda $<1$ um) that can be located anywhere in a patrol field with a radius of 1 arcmin around the LGS. The maximum zenith distance required for this mode is $60 \mathrm{deg}$.

2. SCAO NGS mode in the visible (lambda $<1$ um) with a reference star that can be located on-axis or up to 1 arcmin off-axis. The maximum zenith distance required for this mode is $70 \mathrm{deg}$.

3. Seeing Enhancer mode: SCAO LGS with (any) one of the 4LGSF laser on-axis and no tilt star. Only the HO are corrected. It is a subset of the SCAO LGS mode, where the Active Optics Guider is used for tracking the TipTilt.

4. Seeing limited mode, for which the AO is not operating. In this case, the AO design has to guarantee that the nooperating state is not preventing seeing limited observations.

\section{SPIFFIER: NIR INTEGRAL FIELD SPECTROMETER}

SPIFFIER (SPectrometer for Infrared Faint Field Imaging with Enhanced Resolution) is a near-infrared integral field spectrograph that operates between $1.08-2.43 \mu \mathrm{m}$. The spectrograph operates with 4 gratings (J, H, K, high-res (JHK)). SPIFFIER will use a slicer that divides the sky field of view into 32 slitlets. Each one of the slitlets is imaged onto 64 pixels of the detector, getting then 2048 spectra of the image region. Pre-optics allows choosing the angular size of the slices on the sky. The choices are 250mas, 100 mas and $25 \mathrm{mas}$, leading to field of views on the sky of 8 "x $8 ", 3.2 " x 3.2 "$ and 0.8 "x 0.8 " respectively.

The summary of the changes from SPIFFI to SPIFFIER are: the new pre-optics collimator, the new detector, new cryogenic motors, the H-resolution grating for $\mathrm{J}+\mathrm{H}+\mathrm{K}$, the change of the grating drive, the new $\mathrm{M} 3$ mirror, the control software, the cold finger, order of the sorting filters, the cryostat extension periscope (cold plate extension), the removal of the sky spider, the infrasil dichroic, and the new cryostat lid, plus all electronics and cabling.

SPIFFIER will also have cold baffles to reduce the straylight input to the cold optical path. The SPIFFI baffles will be remade at MPE to be adapted to a $\mathrm{f}=13.6$ beam and the holes for the sky-spider fields will be removed. The positions of the baffles are on the LN2 shield, the sky-spider housing and at the entrance focal plane itself.

The cryogenic motors will be replaced by PYTHRON motors with an adapted mechanical design to allow them to be used on the same mechanical interface. Additionally, the motors for the filter and the optics-wheel are going to be equipped with cryogenic resolvers from LTN Servotechnick. To prevent problems with the grating drive, a new gear will be implemented together with a stronger Phytron motor.

\subsection{Cryostat}

The cryostat will also be the same as SPIFFI, using the LN2 bath cryostat, with only the change of the lid, and will include a new flat IR transparent entrance window $\left(\mathrm{CaF}_{2}\right)$ of $26 \mathrm{~mm}$ diameter and $6 \mathrm{~mm}$ thickness, that will have a cold surface behind it to limit the thermal background. The design requires a new lid that will be made at MPE or a sub-contractor to MPE.

All cryo-vacuum performance must be at least the same as SPIFFI. The Vacuum hold-time of the cryostat when cold shall be longer than 3 weeks without pumping. The design goal hold time for liquid nitrogen of the cryostat shall be 39 hours. The vacuum pressure of the SPIFFIER cryostat shall be less than $10^{-4} \mathrm{mbar}$ when the cryostat is at ambient temperature. The vacuum pressure of the cryostat shall be less than $10^{-6} \mathrm{mbar}$ when the cryostat is at cryogenic temperature. 

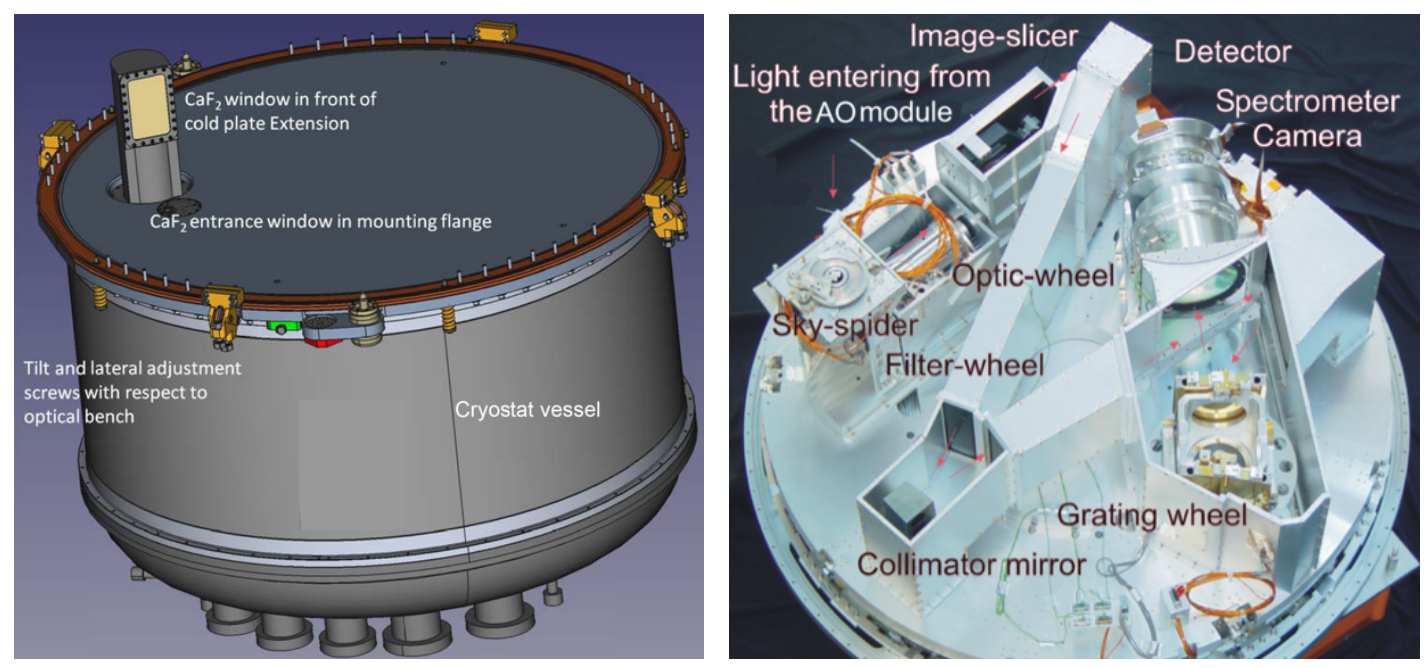

Figure 7. The SPIFFIER cryostat with the entrance window at the left. The inside of SPIFFI: the same design will be used in SPIFFIER, but with new and improved components.

\subsection{Collimator}

The preoptics collimator needs to be changed because of the changed input focal ratio (from $f=17.1$ to $f=13.6$ ) of the telescope beam, and consists of the same 2 lenses of the SPIFFI collimator plus a single meniscus lens (made of $\mathrm{BaF}_{2}$ ) close to the entrance focal plane that adapts the collimator focal length to keep the pre-optics pixel scale constant while preserving the pupil. The old lenses will be replaced by new $\mathrm{BaF}_{2}$ and IRG2 lenses with the same coatings used for the lenses during the early SPIFFI upgrade (2016). It also has a fold mirror, that is a commercial gold-coated 1-inch device.

\subsection{Filter Wheel}

The high-resolution grating will require a set of order sorting filters to avoid mixing of grating orders. Two to three extra order sorting filters will be required for J-band, two for H-band, and none for K-band. The filter wheel has 18 positions, but only 5 slots will be used in regular basis, the filters for: $\mathrm{J}, \mathrm{H}, \mathrm{K}$, open and close.

\subsection{Optic wheel}

Below the entrance focal plane baffle, a triplet lens unit collimates the light onto a cold stop for the suppression of the thermal background. After the cold stop, the motorized optics wheel provides the interchangeable lens systems for the three different image scales (250, 100 and 25 mas), and a pupil imaging lens for alignment purposes. Each objective consists of two or three lenses.

\subsection{Image Slicer}

The focus of the pre-optics is located at the small slicer. This part of the image slicer consists of a stack of 32 plane mirrors, which slices the image into slitlets, and separates the light from each slitlet in different directions. A second set of 32 mirrors, the big slicer, collects the light and forms the pseudo-slit, which appears as a "brick-wall pattern" on the detector. To avoid differential thermal contraction, the unit is completely made from a zero-expansion glass, and all parts are optically contacted.

\subsection{Spectrometer Collimator}

After the image has been sliced and re-arranged into the pseudo-slit, three diamond turned mirrors collimate the light onto the gratings. The first mirror is spherical, and the other two mirrors have an oblate elliptical shape. All mirrors are made from Aluminium. The mirrors are gold-coated for higher reflectivity.

\subsection{Grating}

The spectrograph operates with 4 gratings $(\mathrm{J}, \mathrm{H}, \mathrm{K}$ and High-res $(\mathrm{J}, \mathrm{H}, \mathrm{K}))$ providing a spectral resolution around 2000, 3000,4000 and 8000 (TBC) respectively. The J, H, K will fit fully onto the 2048 pixels, but the high-res will fit only half of the spectral band onto the 2048 pixels. 
The design of the SPIFFIER high resolution grating $(\mathrm{J}+\mathrm{H}+\mathrm{K})$, consist in a reflection gold coating grating, that has to work at low temperatures $(\sim 80 \mathrm{~K})$. To achieve a resolution of 8000 , the high-res (HR) grating needs to be tilted by a large angle (see Fig 8, right) w.r.t. the incoming beam, but generates a vignetting on the camera front lens and fold mirror (6\%).

To avoid the Coefficient of Thermal Expansion (CTE) mismatch in bimetallic structures, like the grating blanks and the $\mathrm{NiP}$ coating, the new gratings will use blanks made by Zerodur, but this will require an isostatic mounting.

It still under evaluation if the nominal $\mathrm{J}, \mathrm{H}, \mathrm{K}$ gratings will be replaced or not.
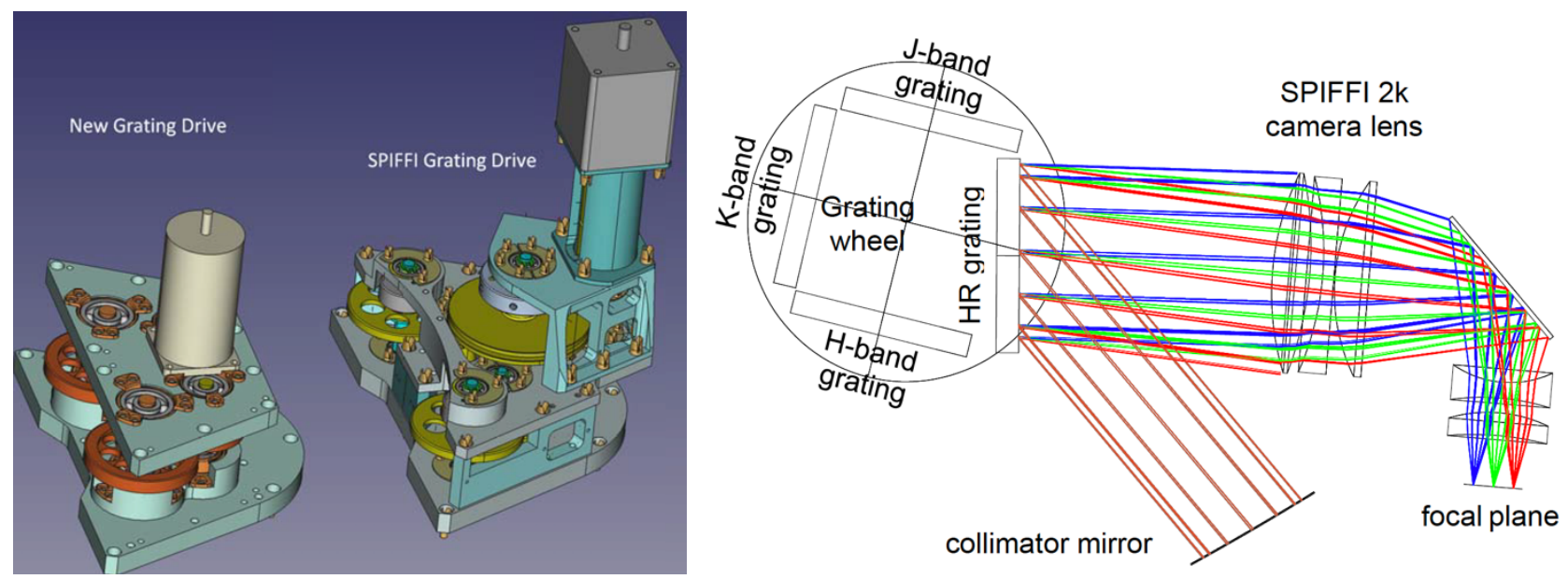

Figure 8 . The new grating drive to the left, and the optical assembly with the HR grating of the grating to the right.

\subsection{Spectrometer Camera}

The camera is a five-lens +1 folding mirror, in a $\mathrm{f} / \mathrm{D}=1 / 2.8$ design. The diameter of the three big lenses is $170 \mathrm{~mm}$. All lenses have a multi-layer anti-reflection coating optimized for the wavelength range from 1.05-2.45 $\mathrm{m}$.

\subsection{Detector}

SPIFFIER will have a new Teledyne H2RG detector, $2 \mathrm{kx} 2 \mathrm{k}$, with the substrate removed (to avoid fringing) and it will be tested by ESO and delivered for the integration.

The actual detector in SPIFFI has in principle a QE of $83 \%$ in $\mathrm{J}$ and $\mathrm{K}$, as measured by the company. The new generation of the Teledyne H2RG detector in principle could have a QE of $90 \%$, with also a lower read noise, but they still have persistence issues. The operational temperature for the detector will be approximately $78 \mathrm{~K}$, since it is cooled with liquid nitrogen as with SPIFFI. The cold finger will be replaced to match the new detector.

The Detector control electronics follows ESO standard, but the cryogenic preamplifier and the PCB cables will stay the same as for SPIFFI.

The cryogenic preamplifier and the flexi PCB cables will stay the same and be reused. The main changes required to go from IRACE to NGC will be the use of new warm cables, those that go from the hermetic connectors to the front panel of NGC. These will be specific cables, designed for this interface only.

\section{NIX: NIR IMAGING CAMERA}

The infrared camera NIX is a subsystem of ERIS. The instrument optics is designed to have pixel scales of 13 mas/pixel $(1.1-2.5 \mu \mathrm{m})$ and $27 \mathrm{mas} /$ pixel $(1.1-5.4 \mu \mathrm{m})$ provided by the camera wheel mechanism.

It will provide diffraction-limited imaging, sparse-aperture masking (SAM) and pupil plane coronagraphy capabilities in the $1-5 \mu \mathrm{m}$ (J to M') bands, either in "standard" observing mode or with "pupil tracking" and "cube" readout mode. The camera optics is able to provide a Field-of-View $(\mathrm{FoV})$ of 24 " $\times 24$ " in the $\mathrm{J}$ to Ks bands, or 53 " $\times 53$ " in the $\mathrm{J}$ to $\mathrm{M}$ ' bands. 


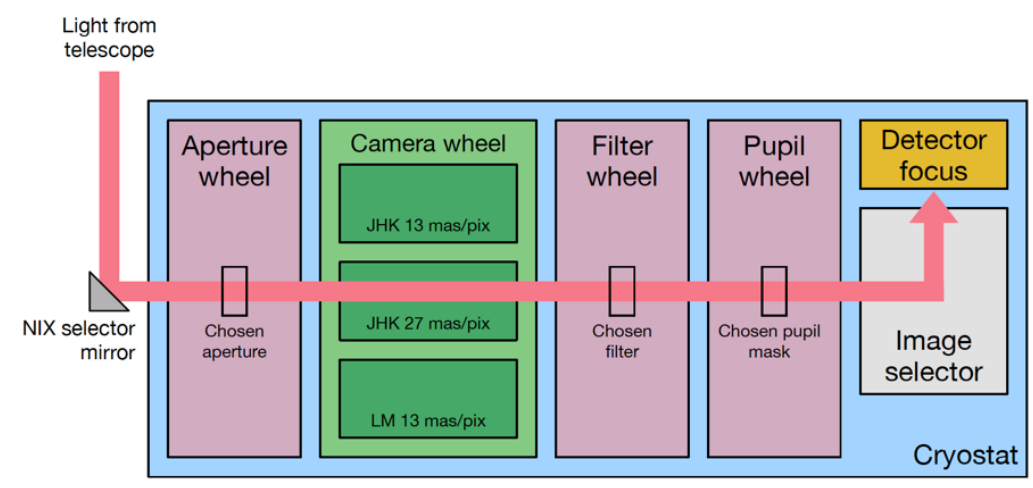

Figure 9. NIX mechanism layout.

\subsection{Cryostat}

The NIX cryostat is designed to be a stand-alone sub-assembly of the ERIS instrument. It has its own vacuum, cooling and electrical systems and operates independently of SPIFFIER.

The NIX cryostat is an Aluminium vessel, machined from solid billets of 6082-T6. The Closed Cycle Cooler (CCC) will be procured from Oerlikon Leybold Vacuum, with an anti-vibration mount manufactured by UK ATC. ESO will provide an anti-vibration mounting assembly for the CC compressor similar at the one used in KMOS. The optics and mechanical structure inside the cryostat will be around $80 \mathrm{~K}$ allowing a minimal instrumental thermal background for the longer wavelengths bands K, L, M. The motors to control the filter, aperture and pupil wheel are going to be Phytron stepper motors, with LTN resolvers. The temperature sensors will be also Phytron PT100.

The light from the NIX selector mirror enters the NIX cryostat via a window in the vessel lid. This window is flat glass and will remain at ambient temperatures.

\subsection{Imaging Cameras}

The detector will be a Teledyne HAWAII-2RG $5.3 \mu \mathrm{m}$ cutoff material, 2048x2048 pixels, operated at $40 \mathrm{~K}$ by a close cycle cooler and controlled by a standard NGC. The substrate will be removed as in SPIFFIER. The dark current measured on similar detectors is typically $0.01 \mathrm{e} / \mathrm{pixel} / \mathrm{second}$. Working at that temperature, the cosmetics of the detector will improve. The detector readout noise shall be less than 15 (goal 10) e rms in Correlated Double Sampling mode.

It will also use the new version of the cryogenic preamplifier, which allows pixel read rates in excess of $1 \mathrm{Mpixel} / \mathrm{second}$. This means that the detector will have high pixel rate/low pixel rate readout mode available to the user (programmable), but having in mind that a consequence of the high pixel rate increase the read noise.

The Detector control electronics follows ESO standard, with a cryogenic preamplifier mounted close to the detector, plus the NGC system, that digitalizes the video signals in parallel, and then transmits the final digital values down an optical fiber to the LCU. The NIX camera is required to be operated synchronised to the secondary mirror when it is chopping.

The optical system comprises three imaging cameras, providing different plate scales and the pupil imaging system. Different field stops are required for the different plate scales; these are interchangeable by means of a deployment mechanism.

The light beam then passes through a set of camera lenses. The lenses for the three cameras are mounted in a camera barrel mechanism (camera wheel) and are interchangeable.

The pupil imager system comprises a pair of ZnSe lenses, which are deployed in the space between the PFW mechanism (pupil and filter wheel) and the deployable fold mirror. They form an image of the cold stop on the detector with a magnification of $\sim 2.28$.

\subsection{Pupil wheel}

The pupil wheel will hold a grism for spectroscopy, dedicated pupil masks and coronagraph mask onto the free slots. It can house up to 20 elements, as the filter wheel. 
The masks are thin plates made from Molybdenum and blackened with Acktar Fractal black coating. There will be 2 reduced pupil masks (JHK, LM), the spider mask, 2 APP (Apodizing Phase Plates) masks (180, 360) and 3 SAM (Sparse Aperture Mask) masks (23, 9, 7 holes), plus the Lyot Stop and a block, for a total of 10 masks.

The grating vector APP coronagraph is a single optic that sits in the pupil plane of the telescope. It adds a phase pattern across the wavefront of the whole telescope pupil. The resultant PSF is reshaped so that diffraction around 180 (or 360) degrees of the star is suppressed in a defined region and down to a defined contrast as measured from the peak of the Airy core of the PSF.

The Lyot stop blocks the star light in the pupil plane that is scattered from the secondary obscuration and the secondary support structure, which appears as a monotonically decreasing flux moving away from the structures. The Lyot stop therefore shrinks the perimeter of the current cold stop in order to mask out the scattered on-axis light.

\subsection{Filters wheel}

The filters are nominally $8 \mathrm{~mm}$ thick, Calcium Fluoride. The filters require to be tilted by $\sim 4^{\circ}$ to reduce ghost imaging without affecting the optical imaging performance. The set of filters comprises 7 broadband filters, 9 narrow band filters, one neutral density filter and a short pass (LM blocking) filter. The broadband and narrow band filters are band pass filters that transmit over a defined wavelength range and block other wavelengths. In total 18 imaging filters will be placed in the filter wheel.

The filters will be circular with an outer diameter of $21 \mathrm{~mm}$, and with an optical quality and spectral performance over a clear aperture of diameter $>19 \mathrm{~mm}$.

\subsection{Aperture wheel}

The aperture wheel will contain a block, plus the following elements:

- $\quad$ NIX has two field masks for imaging, both providing square fields for both field of view on sky.

- The 12 arcsec long slit, that in the narrow dimension width is set to allow the required spectral coverage and resolution. Also note that this slit width, which corresponds to 86 mas on sky, is less that the FWHM maximum of an object's PSF in the L band.

- The distortion mask is used to assess any large optical distortion with NIX, it will allow assessment of image quality of the cameras and assist measurement of any tilt on the detectors. The mask will consist of a plate drilled with a grid of holes. The pitch of these holes has been chosen to ensure that at least a $5 \times 5$ grid of holes is visible in both the different camera scales. The holes define a 9x9 pinhole grid, with spacing of $3.426 \mathrm{~mm}(6.4 \mathrm{arcsec})$ spacing. A pinhole of $10 \mu \mathrm{m}$ provides a diffraction limited spot in the $\mathrm{K}$ band.

- 2 Vortex mask, one for L-band and one for M-band.

The image selector has four positions, one for each of the two field sizes, and one to allow the pupil to be imaged. It can also be put in a block position to completely stop light reaching the detector, which is used both for dark calibration of the detector, but also during set up of the instrument to avoid that unwanted light reaches the detector as the correct positions of the other wheels is being selected.

\section{CALIBRATION UNIT}

The calibration unit will provide internal sources for flat fielding and wavelength calibration from J-band to K-band, as well as optical to infrared point sources for AO calibration, including non-common path aberrations to the instrument detector focal planes.

In particular, the ERIS-CU has to provide:

- $\quad$ Photometric flat fields for NIX, in broad- (J, H, K) and narrow-bands;

- $\quad$ Spectroscopic flat fields for SPIFFIER over its full wavelength range $(1-2.5 \mu \mathrm{m})$;

- Wavelength calibration for SPIFFIER, in the same range as above;

- Point-like (DL) and extended (0.5", 1.0" and 1.5") sources over the whole $0.4-2.4 \mu \mathrm{m}$ wavelength range. These sources are used for AO LGS and NGS WFS calibrations and technical checks (e.g. Non-Common Path Aberrations, Differential Flexures between science instruments and AO systems), at optical wavelengths (Rband), and by NIX and SPIFFIER calibrations and technical checks at infrared wavelengths (J, $\mathrm{H}$ and $\mathrm{K}$ bands); 
- Two sets of illuminated slits (3 slits per set) for SPIFFIER IFS internal alignment check (North-South test) at its two largest plate scales, in $\mathrm{J}, \mathrm{H}$ and $\mathrm{K}$ band.

The higher wavelengths (L, M' bands) are not foreseen for the CU. At these wavelengths, calibrations shall be made using on-sky sources (NIX).
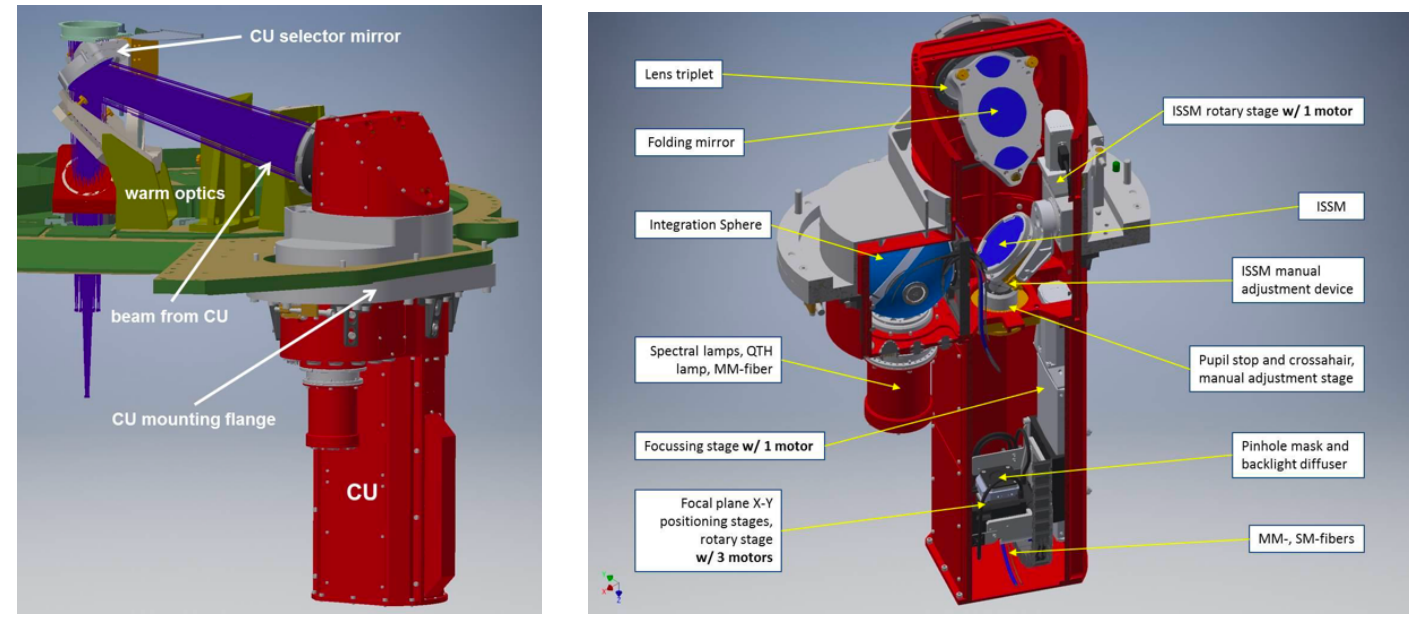

Figure 10. Left: CUMB-CUSM operation, right: CUMB overall view. Both are provided with mechanical adjustment systems in order to accurately align them to the instruments focal planes and pupil planes.

This module is composed by the two following main units.

\subsection{Calibration Unit Main bench (CUMB)}

The Calibration Unit Main bench is optically and mechanically interfaced with the ERIS optical plate. It is equipped with an objective transmitting from visible to $2.5 \mu \mathrm{m}$ with a magnification of 1:1.296 in order to project point sources of various sizes onto the focal plane.

A pattern of artificial point-like sources, extended sources and slits (pinhole mask) is placed onto the back focal plane of the objective. It will provide extremely accurate positioning of sources and slits over the focal plane, as well as to rotate the full pattern with 0.2 arcsec of angular accuracy. The plane can be placed at variable distances from the objective in order to reproduce either celestial sources or laser guide stars. The two modes defined for the CU:

Pupil mode: Flat field capabilities are obtained by inserting a plane mirror across the optical path, in order to project onto the telescope focal plane, the light coming from an integrating sphere. Here is used a diaphragm just in front of the IS to simulate the VLT telescope pupil. The triplet places the image of this diaphragm at the same location of the telescope exit pupil. A fixed pupil stop in the pinhole mask optical path and a folding mirror are also part of the complete CU optical bench.

Focus mode: Sources and slits in the pinhole mask (PHM), placed at the conjugate image plane of the VLT focus, are back-illuminated by rear-feeding the mask with a mono-mode optical fiber (for the DL sources) and a multi-mode optical fiber (for all other sources). The integrating sphere (IS) of 6-inch diameter from Newport, is fed by four pencil-style spectral calibration lamps ( $\mathrm{Ne}, \mathrm{Xe}, \mathrm{Ar}, \mathrm{Kr}$ ) and a QTH continuum lamp, mounted inside a light pipe directly connected to the sphere, plus a multi-mode optical fiber. The sources can move axially to simulate either NGS or LGS.

\subsection{Calibration Unit Fiber Switchyard (CUFS)}

The Calibration Unit Fiber Switchyard, is hosted inside one of the ERIS cabinets and interfaced only with the CU. It contains a Laser Driven Light Source (LDLS), used for flat fields and pinhole mask sources.

This white-light, broad-spectrum lamp provides an almost flat spectral output over the $0.4-2.4 \mu \mathrm{m}$ wavelength range, delivering a power of $50 \mu \mathrm{W} / \mathrm{nm}$ at optical wavelengths and up to $10 \mu \mathrm{W} / \mathrm{nm}$ at $2.5 \mu \mathrm{m}$. 
The LDLS output is sent to a fiber selector placed inside the CUFS. The fiber selector allows to alternatively feed one of the five fibers. In order to adjust the light levels according to the different calibrations to be made, a variable neutral density filter (NDF) can be placed at different positions between the output and the input collimators.

The five input fibers are mounted onto a linear stage, able to provide a positioning accuracy of $1 \mu \mathrm{m}$ over a $155 \mathrm{~mm}$ range. A second identical stage is used for the variable NDF positioning, meeting the same requirements on terms of travel range and positioning accuracy.

\section{CONCLUSIONS}

SINFONI and NACO are two very successful instruments of the first generation at the Paranal Observatory. But despite their key scientific achievements, instruments that have started their operations more than 10 years ago suffer from aging of their components and the limitations of the old technology they were based on. So, should old instruments just be retired while the sciences cases they address are still of high interest? What we demonstrated here with the ERIS instrument is that one can built up on the design of old instruments, using modern technologies, and give them a second life. The interests of this approach are multiple: shorter development time, reduced costs, ensuring continuity in the scientific data.

ERIS is developed based on the design of SINFONI and will re-use its main parts. It thus benefits from the deep study performed on the aging of the optics and mechanical components. If the design is maintained, elements like motorised functions and detectors are being replaced with state of the art systems, therefore improving the performances. The system design is modular and allows access to individual sub-systems by specific handling tools. The instrument has reached compliance to all requirements and already passed the Final Design Review (FDR) stage.

ERIS is also taking advantages of the latest developments in the field of Adaptive Optics, and will benefit from the Adaptive Optics Facility systems implemented on the UT4 at Paranal. This will provide access to a deformable secondary mirror and powerful laser guide stars, that are currently being commissioned and will thus be fully validated and ready for use by ERIS when it will be installed in 2019.

\section{REFERENCES}

[1] Briguglio, R., Biasi, R., et al, “The deformable secondary mirror of VLT: final electromechanical and optical acceptance test results", Proc. SPIE Vol. 9148, 914845 (2014).

[2] Kolb, J., et al, "AOF: first on-sky performance of the GALACSI GLAO mode" AO4ELT5 conference (2017).

[3] Bonaccini, D., Guidolin, I., Friedenauer, A., et al, "The ESO transportable LGS Unit for measurements of the LGS photon return and other experiments", Proc. SPIE 8450, Modern Technologies in Space and Ground-based Telescopes and Instrumentation II, 84501R (2012); doi: 10.1117/12.926898

[4] Bonaccini, D., Hackenberg, W., "The Four-Laser Guide Star Facility: Design considerations and system implementation", Advanced Optical Technologies, ISSN (Online) 2192-8584, ISSN (Print) 2192-8576, DOI: https://doi.org/10.1515/aot-20140025 (2014).

[5] Riccardi, A, Esposito, S., et al, “The ERIS adaptive optics system”, Proc. SPIE 9909, Adaptive Optics Systems V, 99091B (26 July 2016); doi: 10.1117/12.2234001.

[6] Pearson, D., Taylor, W., et al, "NIX, the imager for ERIS: the AO instrument for the VLT", Proc. SPIE 9908, 99083F (9 August 2016); doi:10.1117/12.2234074.

[7] Dolci, M., Valentini, A., et al, "Design of the ERIS calibration unit", Proc. SPIE 9908 , 990839 (9 August 2016 ); doi:10.1117/12.2233662.

[8] George, E., Gräff, D., et al, "Making SPIFFI SPIFFIER: upgrade of the SPIFFI instrument for use in ERIS and performance analysis from re-commissioning”, Proc. SPIE 9908, 99080G (3 August 2016); doi:10.1117/12.2231285.

[9] George, E., Gräff, D., "Complex spectral line profiles resulting from cryogenic deformation of the SINFONI/SPIFFI diffraction gratings", Journal of Astronomical Telescopes, Instruments, and Systems, 035002 (2017); doi: 10.1117/1.JATIS.3.3.035002 